\title{
"To fix, or not to fix" Biological Specimens at a Multi-User Electron Microscopy Facility
}

DeAna Grant and Tommi White

University of Missouri, United States

Advanced technology has expanded both the availability and quality of ultrastructural study from cells to whole organisms, but the rush for the newest microscope must not ignore the most pivotal steps in the study of ultrastructure: specimen preservation and preparation. Developing techniques in the preservation of biological specimens for electron microscopy has greatly expanded the options available for specimen preparation. Methods now range from traditional aldehyde-based fixation to advanced cryo-preparation with availability ranging widely at multi-user microscopy centers around the world. Advances in cryopreservation allow for ultrastructural preservation at near life-like fidelity in model organisms if performed under optimal conditions. High pressure freezing is the methodology of choice when absolute ultrastructural fidelity is required [1]; however, availability of costly instrumentation, user experience, and constraints of sample collection may prohibit the use of advanced cryo-preparation methods for many experiments.

"It is not the pressure-freezing itself, but the preceding preparation, which is the limiting factor also for this type of cryofixation..." Hans Moor [2]

We will discuss how to determine the best preparation techniques for various specimens including cell monolayers, plant tissues, and animal tissues as well as practical considerations for optimal specimen collection outside of a microscopy facility and its impact on methodology of choice. Methods discussed will include chemical fixation followed by room temperature dehydration and resin embedding, microwave-assisted processing, as well as the gold standard in ultrastructural preservation: high pressure freezing and freeze substitution. The advantages and disadvantages of each method will be outlined as it relates to the practical considerations in the day-to-day operations in a multi-user microscopy center for the analysis of diverse specimens by transmission electron microscopy. Finally, we will discuss differentiating artifact from meaningful results.

\section{References}

[1] McDonald, K. (2007) Cryopreparation methods for electron microscopy of selected model systems. Methods Cell Biology. 79, 23-56.

[2] Moor. H (1987) Theory and practice of high-pressure freezing, in Cryotechniques in Biological Electron Microscopy. 